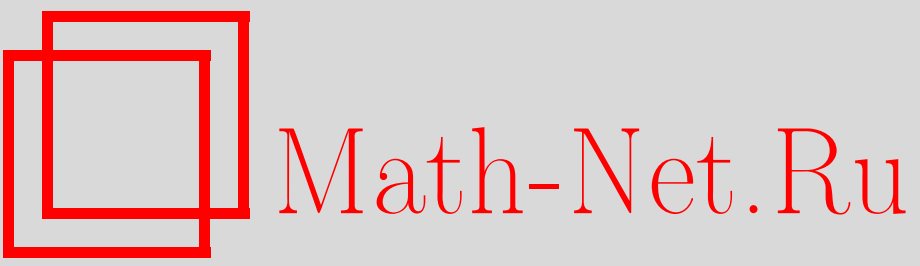

Д. Н. Иванов, Размерность адамаровой алгебры делится на 4, УМН, 2005, том 60, выпуск 2, 163-164

DOI: https://doi.org/10.4213/rm1416

Использование Общероссийского математического портала Math-Net.Ru подразумевает, что вы прочитали и согласны с пользовательским соглашением

http://www.mathnet.ru/rus/agreement

Параметры загрузки:

IP : 35.173 .219 .149

26 апреля 2023 г., 16:33:23 


\title{
РАЗМЕРНОСТЬ АДАМАРОВОЙ АЛГЕБРЫ ДЕЛИТСЯ НА 4
}

\author{
Д. Н. ИвАНОВ
}

Адамаровы алгебры обобщают классические матрицы Адамара ${ }^{1}$, которые отвечают случаю коммутативных алгебр. В статье доказывается, что размерность адамаровой алгебры кратна 4. Это утверждение в качестве гипотезы было впервые сфформулировано в [1].

Напомним основные определения. Пусть $\mathbf{A}$ - ассоциативная конечномерная над полем комплексных чисел $\mathbb{C}$ алгебра, через $\operatorname{Tr}_{A}$ обозначим след регулярного представления $\mathbf{A}$. Тогда $\operatorname{Tr}_{A} x y$ является симметричной инвариантной формой на $\mathbf{A}$, которую мы будем называть формой следа. Известно, что алгебра $\mathbf{A}$ полупроста тогда и только тогда, когда форма следа невырождена на А. Далее будут рассматриваться полупростые алгебры и термин "алгебра", если не оговорено противное, будет обозначать ассоциативную конечномерную над $\mathbb{C}$ полупростую алгебру. Таким образом, по теореме Веддербарна алгебра $\mathbf{A}$ изоморфна конечной прямой сумме матричных алгебр $\mathrm{M}_{n}(\mathbb{C})=\mathrm{M}_{n}$.

ОПредЕЛЕниЕ 1. Семейство неединичных собственных подалгебр $\mathscr{D}=\left\{\mathbf{B}_{i}, i=1, \ldots, r\right\}$ образует ортогональное разложение $(\mathrm{OP})$ алгебры $\mathbf{A}$, если все подалгебры $\mathbf{B}_{i}$ полупросты, содержат единичный элемент $\mathbf{1}_{A}$ алгебры $\mathbf{A}$ и выполняется условие ортогоналшности: алгебра $\mathbf{A}$ является прямой суммой попарно ортогональных подпространств, $\mathbf{A}=\left\langle\mathbf{1}_{A}\right\rangle \oplus \mathbf{B}_{1}^{\circ} \oplus \cdots \oplus \mathbf{B}_{r}^{\circ}$, где $\mathbf{B}_{i}^{\circ}=\left\{x \in \mathbf{B}_{i} \mid \operatorname{Tr}_{A} x=0\right\}$.

Если все подалгебры ОР $\mathscr{D}$ коммутативны, то $\mathscr{D}$ назьвается коммутативным. Если все подалгебры в $\mathscr{D}$ изоморфны алгебре $\mathbf{B}$, то $\mathscr{D}$ назьвается однор одным $O P$ типа $\mathbf{B}$ (или просто $O P$ mипа В). Однородное ОР типа $2 \mathrm{M}_{1}$ назьвается адамаровым разложсением. Алгебра, допускающая адамарово разложение, назьвается адамаров ой. Через $k \mathrm{M}_{n}$ будем обозначать прямую сумму $k$ экземпляров алгебры матриц $\mathrm{M}_{n}$.

В [1] доказана следующая фундаментальная теорема.

Теорема 1 [1]. Алгебра $\mathbf{A}$ является свободныц (левым, правым) модулем над каждой подалгеброй из семейства, образующего коммутативное ОР алгебры $\mathbf{A}$.

Применяя теорему 1 к адамаровым разложениям, которые, очевидно, являются коммутативными, получаем следующую лемму.

Лемма 1. Пусть подалгебры $\left\{\mathbf{B}_{1}, \ldots, \mathbf{B}_{r}\right\}$ образуют адамарово разложение алгебры $\mathbf{A}, \operatorname{dim} \mathbf{A}=n$. Пусть $\operatorname{Pr}\left(\mathbf{B}_{i}\right)=\left\{e_{i 1}, e_{i 2}\right\}-$ примитивнье идемпотенты подалгебрь $\mathbf{B}_{i}$, $i=\overline{1, r}$. В каждой подалгебре $\mathbf{B}_{i}$ вьберем инволюцию $A_{i}: A_{i}^{2}=\mathbf{1}_{A}, A_{i} \neq \mathbf{1}_{A}$. Тогда

(1) $\operatorname{Tr}_{A} e_{i 1}=\operatorname{Tr}_{A} e_{i 2}=n / 2$ для любого $i=\overline{1, r}$, в частности, $n$ четно;

(2) $A_{i}= \pm\left(e_{i 1}-e_{i 2}\right)$, и инволючии $A_{i}$ вместе $с$ единичным әлементом $\mathbf{1}_{A}$ образуют ортогональный базис алгебры $\mathbf{A}$, в частности, $r=n-1$;

(3) для любого $X \in \mathbf{A}$

$$
n \operatorname{Tr}_{A} X^{2}=\sum_{i=1}^{n-1}\left(\operatorname{Tr}_{A} X A_{i}\right)^{2}+\left(\operatorname{Tr}_{A} X\right)^{2} .
$$

Следующая теорема является основной.

Теорема 2. Если алгебра $\mathbf{A}=v \mathrm{M}_{1} \oplus \mathrm{M}_{n_{1}} \oplus \cdots \oplus \mathrm{M}_{n_{k}}$, әде $n_{i}>1$ nри $i=1, \ldots, k$, адамарова, то $n_{1}, \ldots, n_{k}$ четны и размерность алгебры $\mathbf{A}$ делится на 4 . В частности, $v$ делится на 4 .

Работа выполнена при поддержке Российского фонда фундаментальных исследований (грант № 02-01-00219).

${ }^{1}$ Квадратная матрица, состоящая из \pm 1 , назьвается матрицей Адамара, если ее строки, а следовательно, и столбцы, ортогональны. 
ДокАЗАТЕЛЬСтво. Положим $n=\operatorname{dim} \mathbf{A}=v+n_{1}^{2}+\cdots+n_{k}^{2}, \operatorname{Tr}_{A}=\operatorname{Tr}, \operatorname{tr}-$ след матрицы, $E_{s}$ - единичная матрица порядка $s$. Пусть $X=\left(X_{0}, X_{1}, \ldots, X_{k}\right) \in \mathbf{A}$, где $X_{0}=\left(x_{1}, \ldots, x_{v}\right) \in$ $v \mathrm{M}_{1}, X_{i} \in \mathrm{M}_{n_{i}}, i=\overline{1, k}$. Тогда $\operatorname{Tr} X=x_{1}+\cdots+x_{v}+n_{1} \operatorname{tr} X_{1}+\cdots+n_{k} \operatorname{tr} X_{k}$. Пусть подалгебры $\left\{\mathbf{B}_{1}, \ldots, \mathbf{B}_{n-1}\right\}$ образуют адамарово разложение алгебры $\mathbf{A}$. В каждой подалгебре $\mathbf{B}_{s}$ выберем инволюцию $A_{s}=\left(A_{s 0}, A_{s 1}, \ldots, A_{s k}\right)$, где $A_{s 0}=\left(a_{s 1}, \ldots, a_{s v}\right) \in v \mathrm{M}_{1}, A_{s i} \in \mathrm{M}_{n_{i}}$.

Шаг 1. Покажем, что $n_{1}, \ldots, n_{k}$ четны. Подставляя в (1) $X=\left(0, \ldots, 0, E_{n_{i}}, 0, \ldots, 0\right)$, где $E_{n_{i}}$ - единичная матрица в $\mathrm{M}_{n_{i}}$, получим $n n_{i}^{2}=\sum_{s=1}^{n-1}\left(\operatorname{Tr} A_{s i}\right)^{2}+n_{i}^{4}$. Отсюда, после сокращения на $n_{i}^{2}$, вьводим

$$
n-n_{i}^{2}=\sum_{s=1}^{n-1}\left(\operatorname{tr} A_{s i}\right)^{2} .
$$

Так как след инволютивной матрицы есть целое число, то в силу неравенства $n-1>n-n_{i}^{2}$ получаем, что для некоторого $s \operatorname{tr} A_{s i}=0$. Отсюда, в силу того, что слединволютивной матрицы имеет одинаковую четность с ее порядком, получаем, что $n_{i}$ четно.

Шаг 2. Покажем, что $n$ делится на 4 . Так как по лемме $1 \operatorname{dim} \mathbf{A}$ четно, то в силу шага $1 v$ четно. Поэтому считаем далее $v \geqslant 2$. Подставим в $(1) X=\left(\left(x_{1}, \ldots, x_{v}\right), 0, \ldots, 0\right)$ :

$$
n\left(x_{1}^{2}+\cdots+x_{v}^{2}\right)=\sum_{s=1}^{n-1}\left(x_{1} a_{s 1}+\cdots+x_{v} a_{s v}\right)^{2}+\left(x_{1}+\cdots+x_{v}\right)^{2} .
$$

Рассмотрим $((n-1) \times v)$-матрицу $N=\left(a_{s j}\right)$. Из $(2)$ следует

$$
N^{t} N=n E_{n-1}-J_{n-1},
$$

где $J_{n-1}$ - матрица порядка $n-1$, состоящая из одних единиц. Так как $A_{s 0}$ - инволюция, то $a_{s j}= \pm 1$. Заменяя в случае необходимости $A_{s}$ на $-A_{s}$, мы можем без ограничения общности считать, что первый столбец матрицы $N$ состоит из +1 . Если $v=2$, то в силу леммы 1 и шага 1 значение $\operatorname{Tr}$ на примитивных идемпотентах подалгебр $\mathbf{B}_{s}$ нечетно. Значит, $A_{s 0}=(1,-1)$ для всех $s$. Но это противоречит (3). Далее считаем $v \geqslant 4$. Рассмотрим матрицу $N^{\prime}$, образованную первыми тремя столбцами матрицы $N$. Обозначим через $z_{11}, z_{12}, z_{21}, z_{22}$ количество строк в $N^{\prime}$ вида $(1,1,1),(1,1,-1),(1,-1,1),(1,-1,-1)$ соответственно. В силу (3) скалярные произведения столбцов матрицы $N$ (и $N^{\prime}$ ) равны -1 . Следовательно,

$$
z_{11}+z_{12}+1=z_{21}+z_{22}, \quad z_{11}+z_{21}+1=z_{12}+z_{22}, \quad z_{11}+z_{22}+1=z_{12}+z_{21} .
$$

Учитывая равенство $z_{11}+z_{12}+z_{21}+z_{22}=n-1$, находим, что $z_{11}=n / 4-1, z_{12}=z_{21}=$ $z_{22}=n / 4$. В частности, $n$ кратно 4 . Теорема доказана.

ЗАмечАния. 1. В [1] доказано, что алгебра $3 \mathrm{M}_{2}$ не является адамаровой. Таким образом, приведенные в теореме 2 необходимые условия свойства алгебры быть адамаровой не являются достаточными. Однако в случае коммутативных алгебр предполагается, что делимость размерности алгебры на 4 является достаточным условием. Последнее утверждение эквивалентно старой гипотезе о существовании матрицы Адамара любого порядка, кратного 4.

2. Интересна гипотеза: если алгебра $\mathbf{A}=\mathrm{M}_{n_{1}} \oplus \mathrm{M}_{n_{2}}$ адамарова, то $n_{1}=n_{2}$ и любое адамарово разложение алгебры $\mathbf{A}$ содержит ее централњную двумерную подалгебру.

Автор благодарен проф. М.В.Зайцеву и проф̆. А. В. Михалеву за внимание к работе.

\section{СПИСОК ЛИТЕРАТУРЫ}

[1] Д. Н. Иванов // Матем. сб. 1998. Т. 189. № 12. С. 83-102.

Тверской государственный университет

E-mail: dni@tvcom.ru
Представлено А. В. Михалевьм Принято редколлегией 25.01.2005 